\title{
Effect of Radial Transport on the LH Current Drive Efficiency in Tokamaks Featuring an Internal Transport Barrier
}

\author{
L. F. Ziebell ${ }^{1}$, C. I. de Oliveira ${ }^{1}$, and P. R. da S. Rosa ${ }^{2}$ \\ ${ }^{1}$ Instituto de Física, UFRGS, Caixa Postal 15051, 91501-970, Porto Alegre, RS, Brasil \\ ${ }^{2}$ Departamento de Física, UFMS, Caixa Postal 549, 79070-900, Campo Grande, MS, Brazil
}

Received on 26 January, 2004; revised version received on 1 May, 2004

\begin{abstract}
In the present paper we study the effects of occurrence of radial transport of particles in a tokamak, and the effects of the presence of an Internal Transport Barrier (ITB), on the current drive efficiency and power deposition profiles in the case of lower hybrid waves generating an extended tail in the electron distribution function. The results are obtained by numerical solution of the Fokker-Planck equation which rules the evolution of the electron distribution function. We assume that the radial transport of particles is due to magnetic or electrostatic fluctuations, and introduce a model to describe the ITB, with adjustable parameters. The presence of an Edge Transport Barrier (ETB) is simulated by Neumann boundary conditions at the plasma edge. The results obtained show very different behavior for current drive whether we have electrostatic or magnetic transport origin. The change in the plasma current due to magnetic transport has been observed to be more significant than the change due to electrostatic transport, basically because the magnetic transport is more effective in diffusing high-velocity particles of the electron tail.
\end{abstract}

\section{Introduction}

The injection of $\mathrm{rf}$ waves in tokamaks with the objective of current generation has been proposed and studied as an effective way to extend the duration of the tokamak discharge. Lower hybrid (LH) waves play prominent role in the studies related to current generation, either together with other current drive (CD) schemes, or individually. LH waves resonate with electrons via Landau damping, transferring parallel momentum to the electrons, parallel referring to the toroidal magnetic field.

On the other hand, the discovery of an enhanced confinement regime in tokamaks has revealed the existence of Transport Barriers (TB) at the plasma edge (ETB), characterized by steep gradients in electron temperature and density profiles in this region. More recently it has been shown for several tokamaks the presence of Internal Transport Barriers (ITB's) in reversed shear configurations. These ITB's are characterized by a localized and significant reduction of the transport coefficients for heat, particle and momentum. These ITB's appear in plasmas heated by several kinds of heating schemes: ohmic, lower hybrid, ion cyclotron, electron cyclotron, and neutral beam injection. For example, the L-H transition using LH current drive with two different frequencies has been observed by the authors of Ref. [1]. The general mechanism of the formation of ITB's is not well understood but there are evidences that the main mechanism acting to form these barriers in such configurations is the existence of a radial electric field caused by the different responses of electrons and ions to the instability, proportional to the mass ratio between electrons and ions $\left(m_{e} / m_{i}\right)$. In the presence of this electric field the $\mathbf{E} \times \mathbf{B}$ mechanism acts in order to diminish the turbulence in the magnetic surface, therefore lowering the transport between neighborhood surfaces. As a consequence of the formation of the barrier there is accumulation of particles and energy in the region internal to the barrier, with the corresponding increase in the energy confinement time.

In this paper we intend to discuss the effect of different mechanisms causing radial transport, along with the presence of both ITB and ETB, on the LH power absorption and current drive as well on the Electron Cyclotron Emission (ECE). These calculations are made in the frame of the quasilinear theory by solving the Fokker-Planck equation in slab geometry, taking into account corrections both to the density and temperature profiles.

The paper is organized as follows. Section 2 discusses the equations which are employed and the approximations made. Section 3 shows the results found by numerical solution of the Fokker-Planck equation, as a function of several parameters. Due to the complexity of the problem, the numerical investigation is concentrated on the effect of three major variables among the several parameters which have to be assumed, namely the depth of the barrier and the level of magnetic or electrostatic turbulence.

\section{The Fokker-Planck equation and the numerical scheme}

We start with a maxwellian distribution function and solve the Fokker-Planck equation obtained from quasilinear the- 
ory. If we take into account collisions, LH waves and particle transport the Fokker-Planck equation is written symbolically as follows

$$
\partial_{\tau} f=\left(\partial_{\tau} f\right)_{l h}+\left(\partial_{\tau} f\right)_{c o l}+\left(\partial_{\tau} f\right)_{t}
$$

where the subscripts in the right-hand side denote, from the left to the right, the effect of LH waves, collisions and particle transport. $f=f(u, \mu, s, \tau)$ is the electron distribution function, $\tau$ is the time normalized to the collision time at the center of the slab, $u$ is the electron momentum normalized to the central thermal momentum at $\tau=0, \mu=\cos \theta$ is the cosine of the pitch angle $\theta$ and $s=x / a$ is the normalized radial coordinate in the direction perpendicular to the ambient magnetic field $\mathbf{B}_{\mathbf{0}}$, supposed along the $z$ coordinate. The term related to LH waves may be given as follows,

$$
\left(\partial_{\tau} f\right)_{l h}=\partial_{u_{\|}}\left(D_{l h} \partial_{u_{||}} f\right)
$$

where $D_{l h}$ is the diffusion coefficient in momentum space. We consider LH waves continuously injected in the plasma, with fixed power $P_{L H}(a)$ at the edge of the slab. As the waves progress inside the slab, the power dissipation is taken into account. The propagation of the waves is constrained as described in the paper by Dumont et al ([2]), and can be described as follows.

i) The radial component of the wave vector $\left(k_{r}\right)$ must be positive. Using an electrostatic approximation, this means the following, $N_{\|-}<N_{\|}<N_{\|+}$, where

$$
N_{\| \pm}=N_{\| 0}\left[1 \mp \frac{r}{R_{0} q} \times\left(\frac{\epsilon_{\|}}{\epsilon_{\perp}}\right)^{1 / 2}\right]^{-1}
$$

with $\epsilon_{\|} \cong 1-\left(\omega_{p e} / \omega\right)^{2}-\left(\omega_{p i} / \omega\right)^{2}$ and $\epsilon_{\perp} \cong 1+$ $\left(\omega_{p e} / \omega_{c e}\right)^{2}-\left(\omega_{p i} / \omega\right)^{2} ; \omega_{p e, p i}$ is the plasma angular frequency for electrons (ions), $\omega_{c e, c i}$ is the cyclotron angular frequency for electrons (ions), and $\omega$ is the wave angular frequency;

ii) The accessibility condition, defined by the coupling point between the fast and slow modes,

$$
N_{\|}>N_{\| a c}=\frac{\omega_{p e}}{\omega_{c e}}+\left[1+\left(\frac{\omega_{p e}}{\omega_{c e}}\right)^{2}-\left(\frac{\omega_{p i}}{\omega}\right)^{2}\right]^{1 / 2}
$$

iii) An upper limit in $N_{\|}$due to Landau damping, approximately given by

$$
N_{\|}<N_{\| L}=n_{L} / \sqrt{T_{e}}
$$

where $n_{L}$ is around $6-7$ [2]. Several back and forth trips may occur before complete absorption of the waves due to Landau damping, except for very high values of electron temperature and/or of the parallel refraction index at the plasma edge $N_{\| 0}\left(T_{e}^{2} N_{\| 0}>50, T_{e}\right.$ in $\left.\mathrm{keV}\right)$.

These conditions define the range in $N_{\|}$which is available to LH waves, at each position $s$. The limits of this range will be denoted by $N_{2}$ and $N_{1}$. Due to the Landau resonance condition, there is a corresponding range of parallel velocities where the particles are in resonance with the waves. We assume a Gaussian wave spectrum in the propagation domain, given by $S\left(s, \tau, N_{\|}\right)=S_{0} \exp \left(-\left(\frac{N_{\| \mid}-\bar{N}}{\Delta N_{\|}}\right)^{2}\right)$ where $S_{0}$ is the intensity of $\mathrm{LH}$ waves at position $s$ at time $\tau$. This quantity can be obtained considering that, due to the fast movement of the electrons on the magnetic surface, the energy acquired by the electrons when passing through the region affected by the wave is rapidly spread to all the magnetic surface, and the average LH intensity at each magnetic surface can be given by $P_{L H}(s, \tau) /\left(4 \pi^{2} r R\right)$.

The LH diffusion coefficient is proportional to $S\left(s, \tau, N_{\|}\right)$. It can be found in previous works and will not be repeated here [3]. It depends on the perpendicular component of the refraction index $N_{\perp}$, which is obtained from the dispersion relation, for each value of $\omega$ and $N_{\|}$.

The effect of collisions is introduced by the second term in the right-hand side of equation (1). We have used a linearized form which describes the interaction of fast electrons with body electrons and ions, for each slab position.

The last term in the right-hand side of equation (1) describes the transport of particles and has the following general form

$$
\left(\partial_{\tau} f\right)_{t}=\partial_{s}\left(D \partial_{s} f\right)
$$

$D$ being the particle diffusion coefficient, which can be given as follows $[4,5,6]$,

$$
D=D_{m}+D_{e}=\frac{2 \pi q R_{0} c}{\gamma \mu_{e 0}^{1 / 2} \nu_{e 0}}\left(\mid u_{\|} \widetilde{b}^{2}+\frac{\gamma^{2} \mu_{e 0}}{c^{2}\left|u_{\|}\right|} \widetilde{e}^{2}\right)
$$

where $D_{m}$ is the contribution to transport due to magnetic fluctuations, and $D_{e}$ is the contribution to transport when electrostatic fluctuations are present. In this expression $\widetilde{b} \equiv\left(B / B_{0}\right)$ and $\widetilde{e} \equiv\left(E / B_{0}\right)$ give, respectively, the magnetic and electrostatic level of turbulence. $\widetilde{b}_{0}$ is dimensionless, while the units of $\widetilde{e}_{0}$ are $\mathrm{m} / \mathrm{s} . \nu_{e 0}$ is the collision frequency at the plasma center, and $\gamma$ is the relativistic factor. The quantity $q$ is the safety factor, assumed to be constant along the time evolution. This hypothesis is justified since the evolution occurs in the kinetic time scale, while experimental data show that the changes in the $q$ profile occurs in a longer time scale, of order of seconds [7,8].

The ETB is simulated by imposing Neumann boundary conditions on the electron distribution function at plasma edge, i. e. zero particle flux outward [9], while the ITB is simulated by assuming that the level of magnetic (or electrostatic) turbulence is uniformly equal to $\widetilde{b}_{0}$ (or $\widetilde{e}_{0}$ ) everywhere except in the barrier region, where it is reduced down to a minimum value $\alpha \widetilde{b}_{0}$ (or $\alpha \widetilde{e}_{0}$ ). In all calculations which follow, we assume barriers centered at $x_{p}=a / 2$, with half-width $\beta=3 \mathrm{~cm}$. Specifically, we assume a parabolic reduction of the magnetic turbulence level at the barrier position (for $x_{p}-\beta<x<x_{p}+\beta$ ), such that $\widetilde{b}=\widetilde{b}_{0}\left(a_{1}+a_{2} x+a_{3} x^{2}\right)$ and $\widetilde{e}=\widetilde{e}_{0}\left(a_{1}+a_{2} x+a_{3} x^{2}\right)$, where the $a_{i}$ are constants [10].

As boundary conditions of the barrier region we assume $\widetilde{b}, \widetilde{e}=\widetilde{b}_{0}, \widetilde{e}_{0}$ at $x=x_{p} \pm \beta, \widetilde{b}, \widetilde{e}=\alpha \widetilde{b}_{0}, \alpha \widetilde{e}_{0}$, at $x=x_{p}$. The barrier position is chosen in order to be in accordance with experimental results that show barrier location in the interval 
$0.3<|x / a|<0.5$. It is important to remark that the interaction region for $\mathrm{LH}$ waves is inside the region having as boundary the plasma center and the barrier position. Fig. 1a of Ref. [10] shows the profile of magnetic and electrostatic turbulence, for several values of $\alpha$, and $\beta=3 \mathrm{~cm}$. The barrier width and position are in accordance with experimental results [11].

The $q$ profile appearing in Eq. (6) for the transport diffusion coefficient is simulated by a parabolic profile which has its minimum value at the position $x=a / 2$ where the barrier is located (see Fig. 1b of Ref. [10]). This profile intends to simulate the reversed $q$ profiles typically found in situations where there is ITB formation. For all simulations appearing in the present paper the $q$ profile is the same.

The EC radiation emitted perpendicularly to the magnetic field, at the plasma edge, may be given by the following expressions

$$
\begin{aligned}
I_{o}= & \omega_{c}^{2}\left(\frac{2 \pi}{c}\right)^{2} \int_{x_{0}}^{x_{1}} d x \frac{G_{33}\left(\omega, k_{0}^{\prime}\right)}{\left|N_{o}\right|\left|1-\chi_{33}\right|^{2}} \\
& \times \exp \left(-\frac{2 \omega}{c} \int_{x}^{x_{1}} N_{o}^{\prime \prime} d x^{\prime}\right), \\
I_{x}= & \frac{\omega^{2}}{c}\left(\frac{2 \pi}{c}\right)^{2} \int_{x_{0}}^{x_{1}} d x \frac{\left|\epsilon_{11}-i \epsilon_{12}\right|^{2}}{\left|\epsilon_{11}\right|^{2}\left|N_{x}\right|} G_{11}\left(\omega, k_{x}^{\prime}\right) \\
& \times \exp \left(-\frac{2 \omega}{c} \int_{x}^{x_{1}} N_{x}^{\prime \prime} d x^{\prime}\right) .
\end{aligned}
$$

$G_{11}$ and $G_{33}$ are the components of the locally homogeneous current correlation tensor [12].

\section{Numerical results}

We solve numerically the quasilinear equation, Eq. (1), using the ADI method (implicit in alternated directions, [13]), and obtain the time evolution of the electron distribution function. Eq. (1) depends on $u(0 \leq u \leq 12), \mu$ $(-1 \leq \mu \leq 1)$ and $s(-1 \leq s \leq 1)$, and we represent the $(u, \mu, s)$ space by a grid with $151 \times 51 \times 101$ points.

For the magnetic field, initial density and temperature profiles we make use of the following expressions

$$
\begin{aligned}
B_{0}(s) & =B_{0}(0)\left(1+s \frac{a}{R}\right)^{-1} \\
n_{e}(s, \tau & =0)=\left(n_{e 0}-n_{e a}\right)\left(1-s^{2}\right)+n_{e a} \\
T_{e}(s, \tau & =0)=\left(T_{e 0}-T_{e a}\right)\left(1-s^{2}\right)^{2}+T_{e a} .
\end{aligned}
$$

In these expressions the indexes 0 and $a$ indicate, respectively, values taken at the plasma center and plasma edge. At each collision time the density and temperature are updated, directly from the electron distribution function.

As stated before, we assume Neumann boundary conditions at plasma edge, which imply that the total number of particles at the beginning, $N_{0}$, is kept constant along the calculations. This can be easily seen by integrating the transport term of the kinetic equation:

$$
\frac{d N}{d t}=\int_{-1}^{1} d s\left[\frac{\partial f}{\partial t}\right]=\int_{-1}^{1} d s\left[\frac{\partial}{\partial s} D(s) \frac{\partial f}{\partial s}\right]=0
$$

Imposing Neumann boundary conditions, $\frac{\partial f}{\partial x}=0$ at the plasma edge, we guarantee that the number of particles $(N(\tau))$ is constant along time evolution.

For the numerical analysis, we assume the following parameters: $n_{e 0}=6.0 \times 10^{19} \mathrm{~m}^{-3}, T_{e 0}=3.0 \mathrm{keV}$, $B_{0}(0)=2.65 \mathrm{~T}, a=0.2 \mathrm{~m}, R=1.0 \mathrm{~m}$. For the LH waves, we consider $P_{L H}(a, t)=0.5 \mathrm{MW}$, and choose $\bar{N}_{\| 0}=2.3$ and $f_{L H}=3.37 \times 10^{9} \mathrm{~Hz}$, parameters which guarantee total power absorption and which are in the range of parameters used in experiments.

For these parameters, the values of $N_{1}$ and $N_{2}$, which define the range of parallel refraction indexes covered by the LH spectrum, appear in Fig. (1), as a function of position inside the slab.

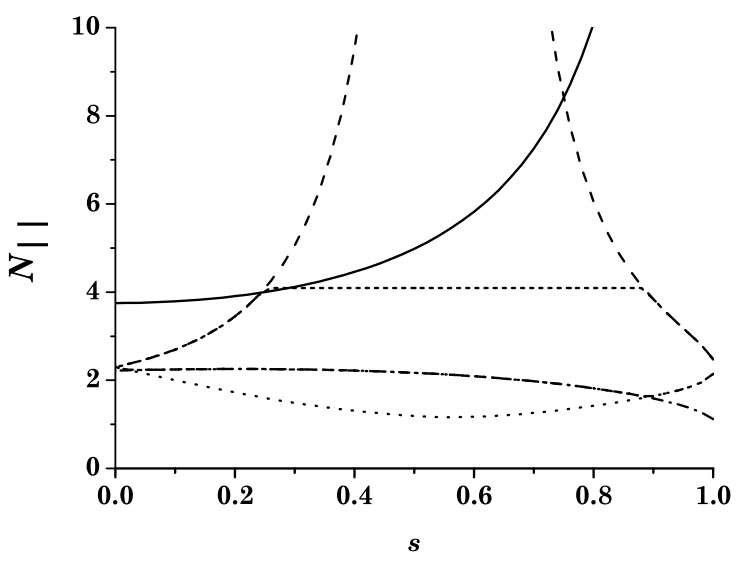

Figure 1. $\quad N_{\|}$accessibility conditions. $\quad N_{\| L}$ (full line), $N_{\|+}$ (dashed line), $N_{\|-}$(dotted line), $N_{\| a c}$ (dashed dotted line), $N_{\| \text {min }}$ (dashed dotted dotted line) and $N_{\| \text {max }}$ (short dashed line). Obs.: the curves for $N_{\| \min }$ and $N_{\| a c}$ are in part superposed.

Figure 2 shows the profile of the density of absorbed $\mathrm{LH}$ power, as a function of electrostatic perturbation level (panel $a$ ) and magnetic perturbation level (panel $b$ ), up to $\tau=50$. The density of absorbed power is obtained as follows (in $\mathrm{W} \mathrm{cm}^{-3}$ ) from $\rho_{l h}(r, \tau)=1.5 \times$ $10^{-16} T_{e 0} \nu_{e 0} \int d^{3} u \frac{u^{2}}{2}\left(\partial_{\tau} f_{e}\right)_{l h}$, where the symbol $(\ldots)_{l h}$ indicates that the integration takes into account the modification in the distribution function caused by the LH term appearing in Eq. (1). It can be seen that the absorption of the LH waves occurs mainly in the vicinity of the magnetic surface with $s=0.5$. The effect of the perturbation level is more important for the magnetic case than for the electrostatic case. This behavior can be explained remarking the different mechanisms for transport in the electrostatic and magnetic cases. While electrostatic transport diffuses mainly particles with small content of parallel momentum the magnetic transport diffuses particles with high momentum content. The LH waves interact with these high energy electrons and thereby as the magnetic transport acts pushing these electrons from the plasma center to the plasma edge 
the absorption of LH waves at more external regions is favoured. However, if we compare the barrier case with the no barrier case (panel $b$ of Fig. 2) the effect of the presence of the barrier appears as a reduction in the LH power absorbed levels outside the barrier region while the values inside the barrier region are greater for the barrier case than for the no barrier case.
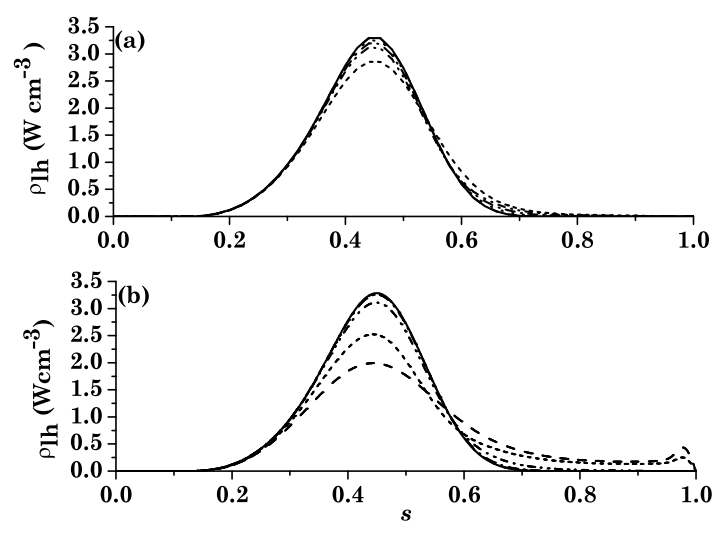

Figure 2. $\rho_{l h}:$ a) $\widetilde{b}=0$ and $\widetilde{e}: 0$ (solid line), $1 \times 10^{2}$ (dashed line), $5 \times 10^{2}$ (dotted line), $7.5 \times 10^{2}$ (dashed dotted line), $1 \times 10^{3}$ (dashed dotted dotted line) and without barrier (short dashed line); b) $\widetilde{e}=0$ and $\widetilde{b}$ : 0 (solid line), $5 \times 10^{-5}$ (dashed line), $1 \times 10^{-4}$ (dotted line), $2.5 \times 10^{-4}$ (dashed dotted line), $5 \times 10^{-4}$ (dashed dotted dotted line) and without barrier (short dashed line). Other parameters are: $\alpha=0.125, s_{b}=0.5, P_{l h}(a)=0.5 \mathrm{MW}$.
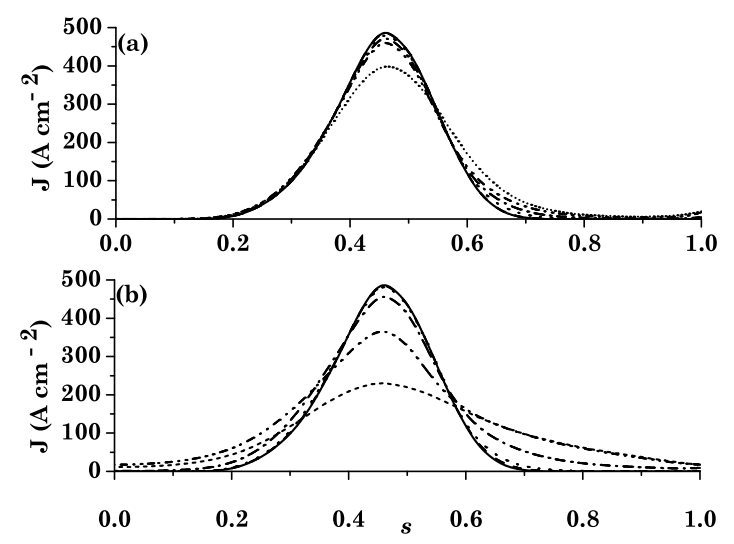

Figure 3. Current density profile: a) $\widetilde{b}=0$ and $\widetilde{e}=: 0$ (solid line), $1 \times 10^{2}$ (dashed line), $5 \times 10^{2}$ (dotted line), $7.5 \times 10^{2}$ (dashed dotted line), $1 \times 10^{3}$ (dashed dotted dotted line) and without barrier (short dashed line); b) $\widetilde{e}=0$ and $\widetilde{b}=: 0$ (solid line), $5 \times 10^{-5}$ (dashed line ), $1 \times 10^{-4}$ (dotted line), $2.5 \times 10^{-4}$ (dashed dotted line), $5 \times 10^{-4}$ (dashed dotted dotted line). Other parameters are: $\alpha=0.125, s_{b}=0.5, P_{l h}(a)=0.5 \mathrm{MW}$. Also shown the case $\alpha=1$ (short dashed line). Other parameters and conventions as in Fig. 2.
The corresponding profile of the current density is shown in Fig. 3. The effects on the current density profile follow the ones in LH power deposition profile. Again the magnetic case is shown to be more sensible to the perturbation level. Comparison between the two panels of Fig. 3 shows the spreading of the current profile due to the transport. However this spread is different for different types of transport. For the electrostatic case the spread due to transport is noticeable only between the plasma edge and the barrier position while for the magnetic transport it occurs both toward the edge and toward the center of the slab, with greater diffusion toward the plasma edge.

The effect of the turbulence level on the current generation is shown in Fig. 4, which displays the time evolution of $I$ as a function of the perturbation level. In both electrostatic and magnetic transport cases we observe an increase on the values of current driven. However, this enhancement of current is much more pronounced in the magnetic case than in the electrostatic case. Again, the explanation is found in the diffusion of high speed particles toward the plasma edge, more pronounced in the case of magnetic transport. This implies that we have more energetic particles in positions before the barrier region and, as LH waves interacts with particles with high parallel momentum content, more LH power is deposited in more external positions. Combined with this we have a lower density region before the barrier region. In this way collisions are less efficient to destroy the created current density. As result of the synergy between these processes the current density in regions near the plasma edge increases and so the current. It's interesting to observe from the panel $(b)$ of Fig. 4 the effect of the barrier as compared with the no barrier case for $\widetilde{b}=5 \times 10^{-4}$. As remarked before, due to the LH power deposition profile behavior, the barrier case shows greater values for the current driven as compared with the case without barrier.

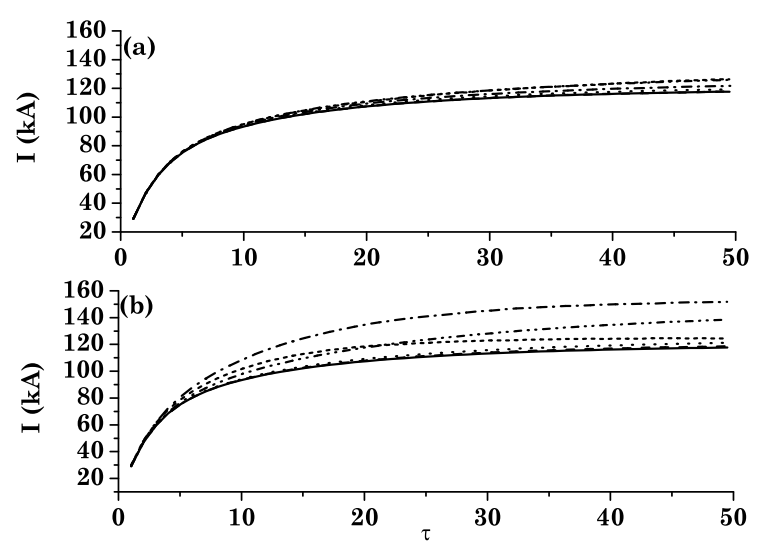

Figure 4. Evolution of the current in time: a) $\widetilde{b}=0$ and $\widetilde{e}=: 0$ (solid line), $1 \times 10^{2}$ (dashed line), $5 \times 10^{2}$ (dotted line), $7.5 \times 10^{2}$ (dashed dotted line), $1 \times 10^{3}$ (dashed dotted dotted line) and without barrier (short dashed line); b) $\widetilde{e}=0$ and $\widetilde{b}=: 0$ (solid line), $5 \times 10^{-5}$ (dashed line ), $1 \times 10^{-4}$ (dotted line), $2.5 \times 10^{-4}$ (dashed dotted dotted line), $5 \times 10^{-4}$ (dashed dotted line). Other parameters are: $\alpha=0.125, s_{b}=0.5, P_{l h}(a)=0.5 \mathrm{MW}$. Also shown the case $\alpha=1$ (short dashed line). Other parameters and conventions as in Fig. 2. 
Similar analysis can be made for the EC emission. Fig. 5 displays the intensity of second harmonic EC emission at $x=a$ as a function of the perturbation level, for the $\mathrm{X}$ mode and perpendicular propagation, at the end of the time evolution, for the same values of $\alpha, \beta$ and $s_{b}$ used in Fig. 2. The figure also shows the emission due to the Maxwellian distribution, prior to the $\mathrm{LH}$ injection. The first thing to observe is the enhancement of the spectral intensity for low frequency values which happens both for the electrostatic and magnetic cases. This enhancement is a direct consequence of the relativistic mass variation of the electrons which permits the electrons to emit at a lower frequency than local EC frequency. As the LH waves push electrons from low momentum to high momentum in momentum space, the emission due to these electrons moves toward smaller frequencies, therefore producing increase of the spectral intensity in the low frequency part of the spectrum.

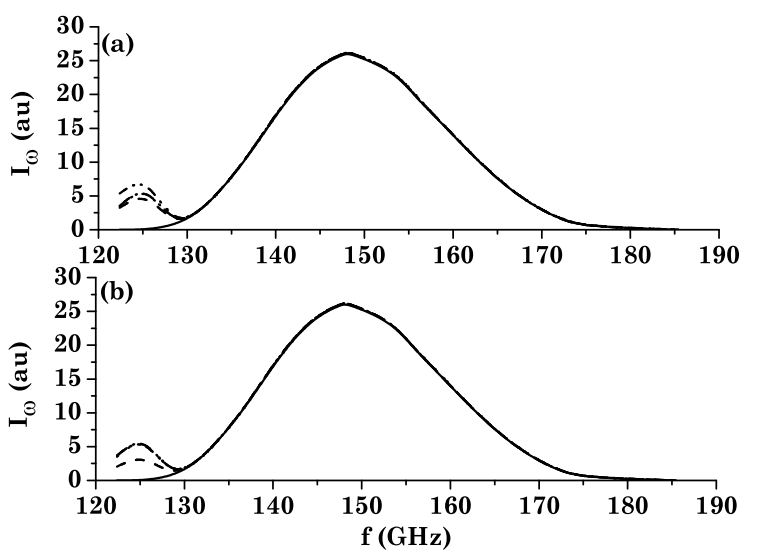

Figure 5. Espectral intensity: a) $\widetilde{b}=0$ and $\widetilde{e}=: 0$ (dashed line), $1 \times 10^{2}$ (dotted line), $5 \times 10^{2}$ (dashed-dotted line), $1 \times 10^{3}$ (dasheddotted-dotted line); b) $\widetilde{e}=0$ and $\widetilde{b}=: 0$ (dashed line ), $5 \times 10^{-5}$ (dotted line), $1 \times 10^{-4}$ (dashed-dotted line), $5 \times 10^{-4}$ (dasheddotted-dotted line) . Other parameters are: $\alpha=0.125, s_{b}=0.5$, $P_{l h}(a)=0.5 \mathrm{MW}$. Also shown the Maxwellian distribution function case (solid line in both panels).

An example of such enhancement may be appreciated in Fig. 6, where the EC emission coefficient $\left(\beta_{x}\right)$ for one of the frequencies in the low part of the spectrum $(f=125.3$ $\mathrm{GHz}$ ) is shown as a function of radial position, at steady state. This frequency corresponds to the second harmonic of the cyclotron frequency near the plasma edge. The emission coefficient appearing in the figure is totally due to the electron tail, since the contribution due to the Maxwellian distribution function at this frequency nearly coincides with the horizontal axis and can not be seen in the scale of the figure. For this frequency the peak in the emission does not occur in the position where this frequency corresponds to the second harmonic, but in the region where the LH waves depose their energy. It is seen from the figure that there are significant differences in the emission profile depending on the type of transport, with the peak in the electrostatic case higher than the peak for magnetic case, almost with the same value of the peak without transport. We attribute this difference to the higher efficiency demonstrated by the magnetic transport to radially diffuse the particles away from the region of creation of the electron tail by the $\mathrm{LH}$ waves, which coincides with the region of the transport barrier. In both cases the emitting region is wider than in the case without transport. This points to lower precision in temperature measures obtained from ECE in such plasmas.

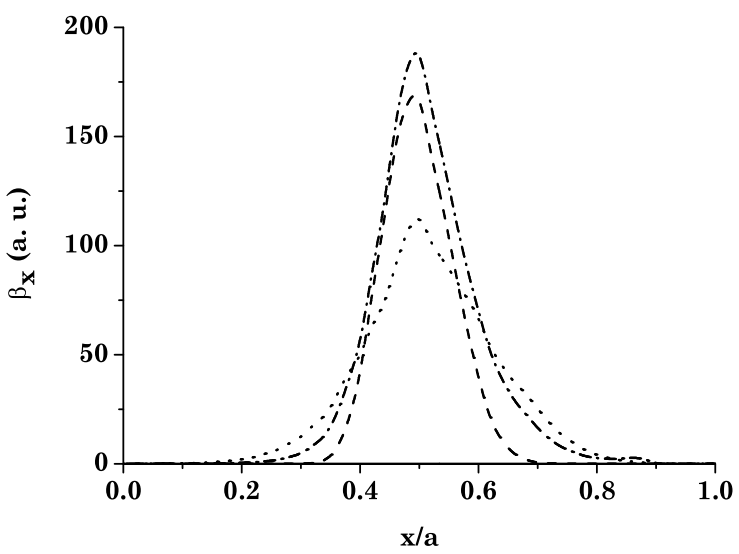

Figure 6. Electron cyclotron emission coefficient for the frequency 125.3 GHz. Maxwellian distribution function (solid line, coincides with the horizontal axis and is not visible in the scale of the figure) and at steady state: Without transport (dashed line), with magnetic transport only (dotted line) and with electrostatic transport only (dahed-dotted line)

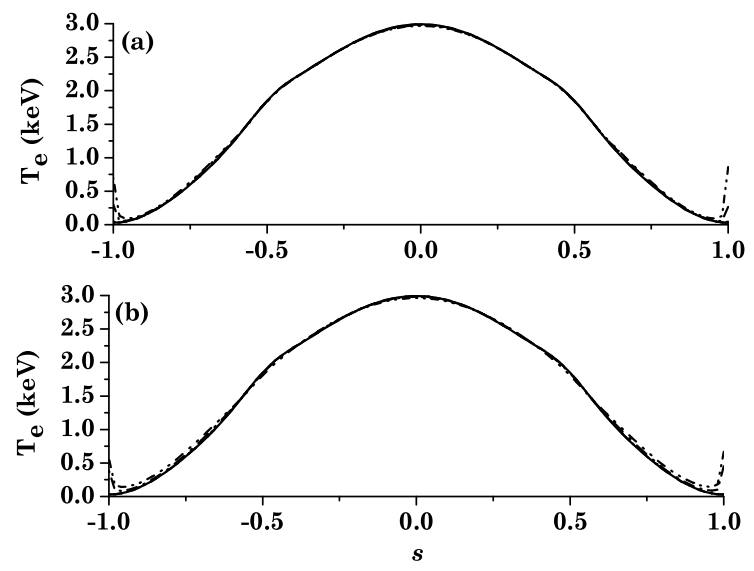

Figure 7. Plasma temperature profile at steady state: a) $\widetilde{b}=0$ and $\widetilde{e}=$ : 0 (solid line), $1 \times 10^{2}$ (dashed line), $5 \times 10^{2}$ (dotted line), $7.5 \times 10^{2}$ (dashed dotted line), $1 \times 10^{3}$ (dashed dotted dotted line); b) $\widetilde{e}=0$ and $\widetilde{b}=: 0$ (solid line), $5 \times 10^{-5}$ (dashed line ), $1 \times 10^{-4}$ (dotted line), $2.5 \times 10^{-4}$ (dashed dotted line), $5 \times 10^{-4}$ (dashed dotted dotted line). Other parameters are: $\alpha=0.125, s_{b}=0.5$, $P_{l h}(a)=0.5 \mathrm{MW}$ 
Figure 7 displays the temperature profile as a function of the perturbation level. It's noticeable the modification in the plasma temperature profile in the region around $s=0.5$, due to the presence of the high energy tail created by $\mathrm{LH}$ waves.

The influence of the barrier depth on the current drive is shown in Fig. 8. The current drive in the electrostatic case is not sensible to the barrier depth as can be seen from the panel $a$ of the figure. However, the current driven in the magnetic case has been shown to be very sensitive to the barrier depth.
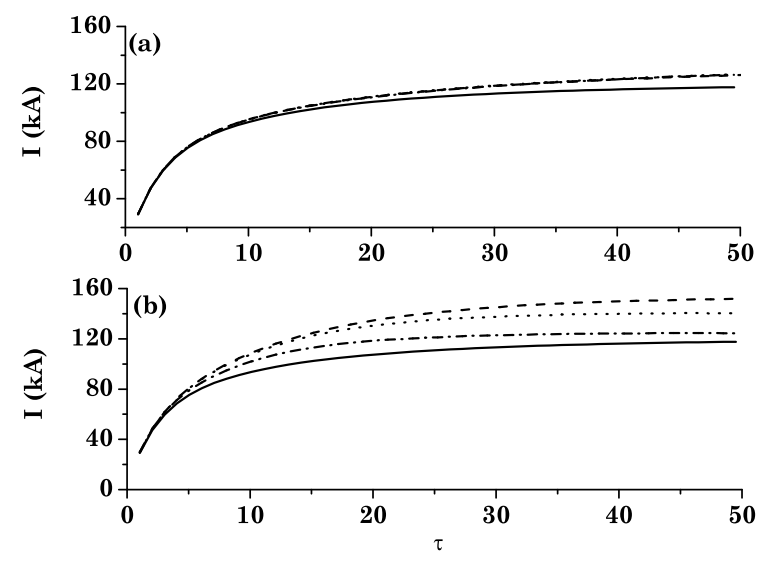

Figure 8. Dependence of the current driven on the barrier depth. a) $\widetilde{e}=1 \times 10^{3}$ and $\widetilde{b}=0$; b) $\widetilde{b}=5 \times 10^{-4}$ and $\widetilde{e}=0$. No transport (solid line), $\alpha: 0.125$ (dashed line), 0.5 (dotted line), and 1. (dashed dotted line). Other parameters and conventions as in Fig. 2.

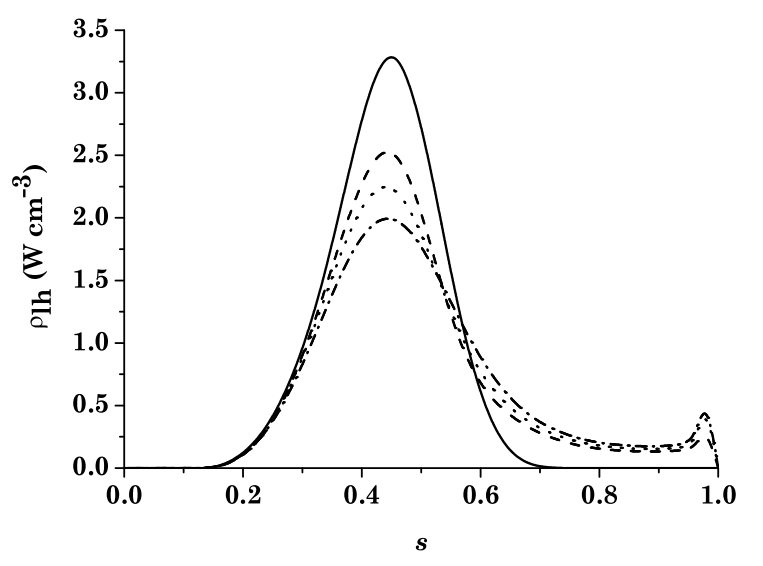

Figure 9. Asymptotic profile of the LH power deposition $\left(\rho_{l h}\right)$ for several values of the barrier depth, in the case of transport due to magnetic fluctuations $\left(\widetilde{b}=5 \times 10^{-4}\right): \alpha=0.125$ (dashed line), $\alpha$ $=0.5$ (dotted line), and $\alpha=1.0$ (dashed-dotted line). The case without transport is also shown, as a solid line. Other parameters and conventions as in Fig. 2.
The reason is the effectiveness of the barrier to constrain the particles inside the barrier region, in this way enhancing the LH power deposition profile as can be seen in the Fig. 9. This is in apparent contradiction with the explanation above about the reason of the increment in the plasma current due to the diffusion of energetic electrons. To understand this behavior we invoke the following argument: the particles inside the barrier region receives energy from the waves until the magnetic diffusion becomes effective and then diffuse toward the plasma edge. As the barrier is stronger (lower $\alpha$ value) the electrons remain more time in interaction with the waves before diffusing. The diffused electrons are therefore more energetic and the value of the current increases.

\section{Conclusions}

In this work we have investigated how the occurrence of radial transport and the presence of an Internal Transport Barrier affect the current drive efficiency of LH waves. We have considered two possible mechanisms for radial transport, either due to magnetic fluctuations or due to electrostatic fluctuations, and studied the influence of the depth of the transport barrier and of the level of magnetic or electrostatic perturbation. For a given type of transport and a given set of parameters describing the barrier, our results have shown increase of the current drive efficiency when the turbulence level is increased, which is explained by considering that the transport diffuses high-velocity particles toward more external regions of the slab, where they are less collisional and contribute more effectively to the current. The increase in the current drive efficiency in the case of transport due to magnetic turbulence has been observed to be more significant than the increase in the case of transport due to electrostatic turbulence. The reason is that magnetic transport is more effective on electrons at the tail of the distribution function, which give the most significant contribution to the current.

Regarding the effect of the depth of the transport barrier, in the electrostatic case, in accordance with the small effect of transport, we have observed very little influence of the barrier depth on the efficiency of the current drive. In the case of magnetic transport, however, we have observed significant increase of the efficiency of current generation, as the depth of the barrier is increased. This increase in the current drive efficiency with the depth of the barrier has been explained by considering that the occurrence of a transport barrier at least partially coincident with the region of deposition of LH power affects the dynamics of tail electrons, by keeping these electrons absorbing wave energy for longer time before diffusing spatially. As a consequence, these electrons become more energetic that in the case without transport barrier, and contribute more effectively to the current when finally diffuse to more external regions of the slab.

This work has been partially supported by Universidade Federal de Mato Grosso do Sul (UFMS), Conselho Nacional de Desenvolvimento Científico e Tecnológico (CNPq), Fundação de Apoio ao Desenvolvimento do Ensino, Ciência e Tecnologia do Estado de Mato Grosso do Sul (FUNDECT) and Fundação para o Amparo da Pesquisa no Estado do Rio 
Grande do Sul (FAPERGS).

\section{References}

[1] S. Tsuji et al., Phys. Rev. Lett. 64, 1023 (1990).

[2] R. Dumont, G. Giruzzi, and E. Barbato, Phys. Plasmas 7, 4972 (2000).

[3] P. R. S. Rosa and L. F. Ziebell, Plasma Phys. Contr. Fusion 41, 525 (1999).

[4] G. Giruzzi, I. Fidone, and X. Garbet, Nuclear Fusion 32, 1011 (1992)

[5] G. Giruzzi, Plasma Phys. Contr. Fusion 35, A123 (1993).

[6] G. Giruzzi et al., Plasma Phys. Contr. Fusion 38, 1593 (1996)
[7] X. Litaudon et al., Plasma Phys. Contr. Fusion 43, 677 (2001).

[8] C. D. Chalis et al., Plasma Phys. Contr. Fusion 43, 861 (2001).

[9] S. J. Wukitch et al., Physics of Plasmas 9, 2149 (2002).

[10] P. R. S. Rosa and L. F. Ziebell, Plasma Phys. Contr. Fusion 44, 2065 (2002).

[11] G. M. Staebler, Plasma Phys. Contr. Fusion 40, 569 (1998).

[12] L. F. Ziebell and D. Dillenburg, Phys. Fluids 26, 80 (1983).

[13] B. Carnahan, H. Luther, and J. Wilkes, 1969. Applied Numerical Methods. John Wiley, New York. 